\title{
The Singularity Problem for Space-Times with Torsion.
}

\section{G. Esposito}

Department of Applied Mathematics and Theoretical Physics

Silver Street, Cambridge CBS 9EW, U.K.

St. John's College - Cambridge CB2 1TP, U.K.

(Nuovo Cimento $B, 105,75$ (1990))

PACS 04.20.Cv - Fundamental problems and general formalism.

PACS 04.50 - Unified field theories and other theories of gravitation.

PACS 99.10 - Errata.

On page 78, the third and fourth lines from the top are erroneous, since it was later found that the unpublished work in ref. [17] was not corret. We are grateful to the authors of ref. [17] for the correspondence about this problem.

\section{Hamiltonian Structure of a Friedmann-Robertson-Walker Universe with Torsion.}

\section{G. Esposito}

Department of Applied Mathematics and Theoretical Physics

Silver Street, Cambridge CBS $9 E W, U . K$.

St. John's College - Cambridge CB2 1TP, U.K.

(Nuovo Cimento B, 104, 199 (1989))

PACS 04.20.Fy - Canonical formalism, Lagrangians, and variational principles.

PACS 04.50 - Unified field theories and other theories of gravitation.

PACS 99.10 - Errata.

On page 207, the third line from the bottom should read:

... (they cannot be brought into the firts class ...) and the bottom line should be deleted.

On page 208, the top line should be deleted, with the exception of the words: "In our notation, the hat" and the third line from the top should read:

... also $\Phi_{2}$ can lead to a differential operator, here denoted by $\hat{\Phi}_{2}$. 